\title{
Two Step Fabrication Of Superhydrophobic Surface On Aluminium With Environmental Stability
}

\author{
Subramanian Sathy Srikandan, \\ \{sss.as@psgtech.ac.in\} \\ Department of Applied Science, PSG College of Technology, Peelamedu, Coimbatore - 641004 ,
}

\begin{abstract}
The unique superhydrophobic property of lotus leaf, encouraged researchers to fabricate artificial superhydrophobic surfaces (SHs) adopting different methods. The purpose of this work is to attempt a modest, robust and environmentally safeprocedure to build a superhydrophobic surface on aluminium substrate by wet-chemical grafting. The substrate after few pretreatments like mechanical, chemical etching, anodizing at different current density and at different time duration was immersed in a solution containing sodium hydroxide in deionized water, lauric acid in ethanol. The effect of duration of anodization and sealing on the production of superhydrophobic surface was highlighed. Using Scanning Electron Micrsocopy (SEM) the surface morphology of the bare aluminium substrate and superhydrophobic surface. Using static water contact angle (WCA) measurements the non-wetting property of the as such fabricated superhydrophboic surface was studied and compared with the bare aluminium substrate. The corrosion resistance of the fabricated superhydrophobic surface in $3.5 \% \mathrm{NaCl}$ medium was examined by potentiodynamic polarization method and compared with the bare aluminium substrate. The fabricated SHs showsvery good corrosion resistance in comparison with the bare aluminiuim substrate in $3.5 \% \mathrm{NaCl}$ medium..
\end{abstract}

Keywords: Fabrication, Environmental, Stability, Surface.

\section{Introduction}

Superhydrophobic materials possess very high water repellency and excellent nonwettability. The water forms a spherical droplets instead of continuous film on the superhydrophobic surfaces.[1, 2]Lotus leaves is one of the very good example which are extremely difficult to wet because of their superhydrophobic nature.[3, 4]In order to achieve the superhydrophobic surface artificially it must have the static water contanct angle greater than $150^{\circ}$ is must and it should possess a very low contact angle hysteresis.[5, 6]

The lotus leaf's superhydrophobic behavior have been referred to as "lotus effect" as shown in Fig. 1(a) and 1(b) respectively. The micro-nano projections present on the lotus leaf is responsible for the superhydrophobic nature.[7, 8] The particles of dirt were rolled over by water droplets due to the micro/nano architecture on the superhydrophobic surface, which is the reason for the minimal droplet's adhesion with the surface.[9] 

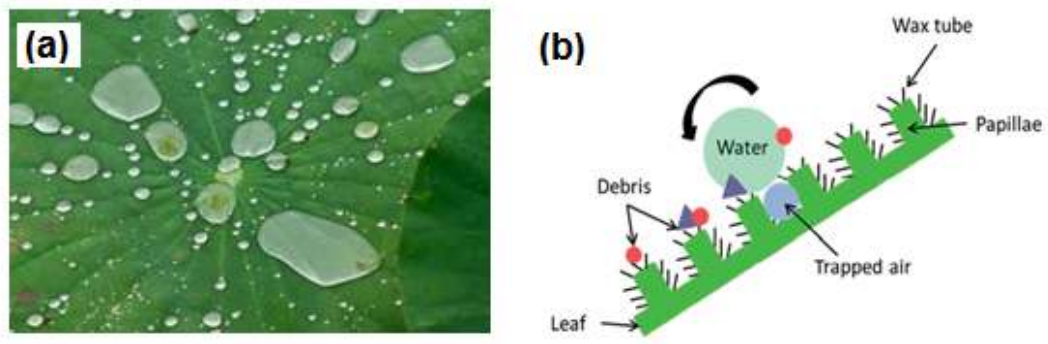
leaf

Figure 1a) Optical image of lotus leaf, b) pictorial representation of self-cleaning of lotus

The unique surface texture and chemistry of the fabricated SHs, controls the wettability. Static water contact angle is measured using the liquid-vapour interface which meets a solid surface.[10,11] The instrument used to measure the liquid solid interface contact angle is named as goniometer. Contact angle $(\theta)$, which is a quantitative measurement of the wetting behaviour of the solid by a liquid. It quantifies the wettabilty of a solid surface by a liquid via the young equation.[12]

1.1 Theoretical background to measure contact angle:

Young's equation is adopted to the smooth surfaces, when a liquid drop is placed on the substrate it comes in contact with a solid, so the equilibrium between the solid and liquid surface will be established at that region the angle between the liquid surface and the solid is termed as contact angle $\mathrm{CA}$ is given by the young equation:-[13,14]

$$
Y \operatorname{CoS} \theta=Y S V_{0}-Y S L-----e q .1
$$

Where, sv, sl and lv are the interfacial surface tensions of the solid-vapour, solid-liquid respectively. (Figure 2a). Young's equation which measures the contact angle $(\Theta)$ is applied only to a flat surface and this equation does not hold good for rough surfaces. Wenzel and Cassie \&Baxtern designed an equation to understand the wetting of rough surfaces.[15]
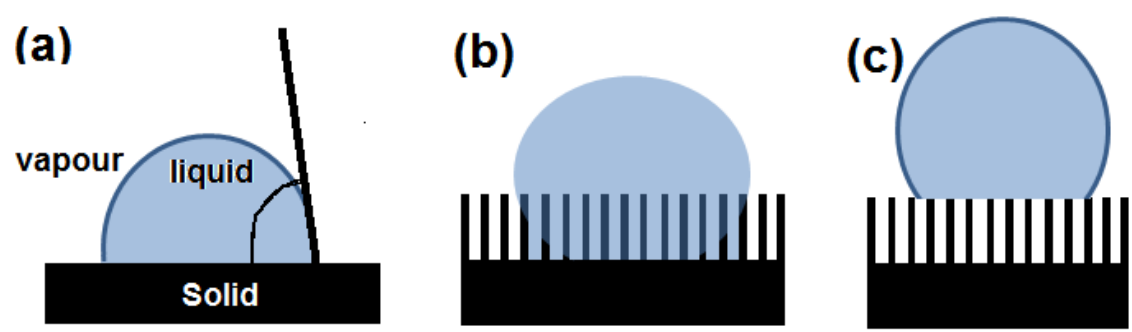

Figure 2: The schematic representation of a liquid droplet on a surface: (a) Young's model (smooth surface), (b) Wenzel's model (rough surface) and (c) Cassie's model (rough surface)

Wenzel modified the young equation. Wenzel model as shown in figure $1 \mathrm{~b}$ is applied, in which the liquid droplet penetrate completely into the rough surface grooves, the contact angle is given by the following equation:[16]

$$
\cos \theta^{*}=r \cos \theta_{Y}----e q .2
$$

Here, $\Theta y$ is the contact angle on a rough surface, and $r$ is the surface roughness factor, which is obtained by finding the ratio between the practically available surface area to the theoretically calculated surface area. The perfectly smooth surface will have the value of $r=1$ 
since the practically available surface area will be equal to the theoretically calculated surface area and $r>1$ for a rough surface in which theoretically available area will be lesser than the actual surface area due to surface imperfections on the rough surface. The air forms a bubble and get trapped on the surface when the value of $\Theta>90^{\circ}$ due to its rough grooves. Practically the surface will be a combination of smooth and rough grooves so a combined Cassie \& Baxter model is always adopted.[17]

Anodizing is an electrochemical process that converts the metal surface into a decorative process in which metals such as $\mathrm{Al}, \mathrm{Ti}, \mathrm{Mg}$ etc., can be anodically oxidized to form a thin oxide layer on their surface. In this process aluminium is made as anode and lead or leadantimony is made as cathode and electrolyzed in electrolyte bath such as $\mathrm{H}_{2} \mathrm{SO}_{4}$, chromic acid, oxalic acid etc., at a specific current density for given period of time, when electrolysis is carried out it result in formation of aluminium oxide layer over the anodic surface, along with evoluation of hydrogen over cathode surface.[18, 19] The following chemical reaction is taking place during the anodization of aluminium substrate using dilute sulphuric acid as electrolyte:

Anode $: A l \rightarrow A l^{3+}+3^{e-}------e q .3$

$$
2 \mathrm{Al}^{3+}+3 \mathrm{O}^{2-} \rightarrow \mathrm{Al}_{2} \mathrm{O}_{3}-------e q .4
$$

Cathode $: 2 \mathrm{H}+2 e^{-} \rightarrow \mathrm{H}_{2}(g)-------e q .5$

Hence overall equation is: $2 \mathrm{Al}+3 \mathrm{H}_{2} \mathrm{O} \rightarrow \mathrm{Al}_{2} \mathrm{O}_{3}-------e q .6$

Although surfaces with superhydrophobicity attract scientists in many aspects of applications, till now the search for excellent superhydrophobic materials is in progress.

A comprehensive list of different methods used to fabricate superhydrophobic surfaces is presented in Table 1.

\begin{tabular}{|c|c|c|c|}
\hline Method & $\begin{array}{l}\text { Interactions between } \\
\text { substrates and coatings }\end{array}$ & Roughness formation & Hydrophobization \\
\hline $\begin{array}{l}\text { Covalent layer-by-layer } \\
\text { assembly }\end{array}$ & Covalent bonding & $\begin{array}{l}\text { Nanoparticle-induced } \\
\text { micro/nanostructure }\end{array}$ & $\begin{array}{l}\text { Using low-surface- } \\
\text { energy chemicals }\end{array}$ \\
\hline $\begin{array}{l}\text { Polymer film } \\
\text { roughening }\end{array}$ & $\begin{array}{l}\text { Polymer film itself as } \\
\text { substrate or } \\
\text { polymer binding }\end{array}$ & $\begin{array}{l}\text { Phase-separation-induced } \\
\text { porous structure of a } \\
\text { multicomponent mixture }\end{array}$ & $\begin{array}{l}\text { Hydrophobic polymer } \\
\text { itself }\end{array}$ \\
\hline $\begin{array}{l}\text { Chemical vapor } \\
\text { deposition }\end{array}$ & $\begin{array}{l}\text { Covalent binding and/or } \\
\text { polymer binding }\end{array}$ & $\begin{array}{l}\text { Growth of nanostructures } \\
\text { by polymerization }\end{array}$ & $\begin{array}{l}\text { Polymerized structure } \\
\text { itself }\end{array}$ \\
\hline Sol-gel process & $\begin{array}{l}\text { Covalent binding } \\
\text { and/or adhesion }\end{array}$ & Rough coating & $\begin{array}{l}\text { Using low-surface- } \\
\text { energy chemicals }\end{array}$ \\
\hline $\begin{array}{l}\text { Hydrothermal } \\
\text { synthesis }\end{array}$ & Covalent binding & $\begin{array}{l}\text { Hydrothermal growth } \\
\text { of nanostructures }\end{array}$ & $\begin{array}{l}\text { Using low-surface- } \\
\text { energy chemicals }\end{array}$ \\
\hline $\begin{array}{l}\text { Coating with } \\
\text { composites of } \\
\text { nanoparticles }\end{array}$ & $\begin{array}{l}\text { Covalent bonding } \\
\text { and/or polymer } \\
\text { binding }\end{array}$ & $\begin{array}{l}\text { Nanoparticle-induced } \\
\text { micro/nanostructure }\end{array}$ & $\begin{array}{l}\text { Using low-surface- } \\
\text { energy chemicals or } \\
\text { hydrophobic polymers }\end{array}$ \\
\hline
\end{tabular}

Table 1: Different methods of fabricating superhydrophobic surface.

\section{Experimental methods}

\subsection{Materials and methods}

Aluminium sheets (1050 grade) were procured from M/s. Coimbatore metal mart, India. The chemical composition were mainly composed of $1.00 \mathrm{wt} . \%$ of $\mathrm{Si}, 1.95 \mathrm{wt} . \%$ of $\mathrm{Mg}, 2.50$ 
$\mathrm{wt} \%$ of $\mathrm{O}$ and $\mathrm{Al}$ balance. All chemicals used were analytical grade purchased from $\mathrm{M} / \mathrm{s}$ Merck India limited, India and used without further purification. Throughout the experiment double distilled water was used.

\subsection{Pretreatment of aluminium sheet}

The aluminium plates $(60 \mathrm{~mm} \times 25 \mathrm{~mm} \times 2 \mathrm{~mm})$ were chemically etched in $5 \mathrm{wt} \% \mathrm{NaOH}$ solution for 5 minutes to remove surface impurities and followed by immersion in $5 \mathrm{wt} \%$ of $\mathrm{HNO}_{3}$ to remove excess alkali stick on the surface of aluminium substrate. The plates were washed well with distilled water and dried with hair drier. The chemically etched aluminium sheets were mechanically polished with \#200, \#400, \#600, \#1200 and \#2000 grades emery sheets and washed well. The mechanically polished aluminum sheets were cleaned with ethanol and followed by water for 15 minutes each in ultrasonic cleaner and air dried.

\subsection{Experimental procedure}

\subsubsection{Preparation of superhydrophobic mixture}

Preparation of superhydrophobic mixture is the important stage of this experiment. To prepare alcoholic solution of lauric acid. About $4.567 \mathrm{~g}$ of lauric acid was taken and dissolved in $100 \mathrm{ml}$ of distilled ethanol at room temperature in a $500 \mathrm{~mL}$ beaker, and sodium hydroxide $(1.501 \mathrm{~g})$ was taken and dissolved in distilled water. The prepared sodium hydroxide was added drop wise into the lauric acid ethanol solution with constant stirring using mechanical stirrer at $298 \pm 5^{\circ} \mathrm{K}$ temperature. After adding sodium hydroxide solution, it is mixed well to obtain a uniform and transparent solution after this it placed in a water bath at $60{ }^{\circ} \mathrm{C}$ for three hours.

\subsubsection{Fabrication of superhydrophobic surface}

The aluminium substrates were immersed in the superhydrophobic mixture kept at $60{ }^{\circ} \mathrm{C}$ for 3 hour, after that the samples were taken out and washed with double distilled water and followed by ethanol to remove un-reacted superhydrophobic mixture. The washed substrates were kept in an hot air oven at $60^{\circ} \mathrm{C}$ for 2 hours which allows the surface adsorbed ethanol on the substrate to volatize slowly. Finally, the samples were taken out of the oven and kept at room temperature.

The same procedure is adopted to three different type of aluminium substrate materials, 1 : Mechanically polished aluminium sheet, (MSH) 2: mechanically and chemically etched aluminiuim, (MCSH) 3: mechanically, chemically polished and anodized at the current density of $6.25 \mathrm{~A} / \mathrm{dm}^{2}$ with $2 \mathrm{M}$ sulphuric acid as electrolyte at different time duration (2 minutes (ASH1), 5 minutes (ASH2), 30 minutes (ASH3) and 60 minutes (ASH4))

\subsubsection{Surface Characterization}

The surface structures of the fabricated substrates were tested under a Scanning Electron Microscope (Joel, Japan). The static water contact angle (WCAs) were obtained from contact angle meter DMs 200 (Kyowa, Japan), The average of WCAs measured at five different places were taken to compare the SHs and bare aluminium substrate. About 4-5( $\mu \mathrm{L})$ volume water droplet were used to measure the WCAs.

\section{Results and Discussion}

\subsection{Wettability}

The wettability of the superhydrophobic surfaces were estimated by measuring the static WCA of the substrate. The WCA was measured by Kyowa 210, japan model static water contact angle meter using senssile drop method. Figure 3 illustrates the water droplet shapes 
on different sample surfaces. Mechanically, chemically polished and anodized surface shows different static WCA. After anodizing the aliminium substrate in $2 \mathrm{M}$ sulphuric acid for 2 minutes at $6.25 \mathrm{~A} / \mathrm{dm}^{2}$, it shows WCAs of $132.3^{\circ}$ Figure $3 \mathrm{a}$. After anodizing the aluminium substrate in $2 \mathrm{M}$ sulphuric acid for 5 minutes it shows WCA of $142.8^{\circ}$ Figure $3 \mathrm{~b}$. After anodizing the aluminium substrate in $2 \mathrm{M}$ sulphuric acid for 30 minutes it shows WCA 157 ${ }^{\circ}$ (for $60 \mathrm{~min}$ ) Figure $3 \mathrm{c}$. In order the get the superhydrophobic surface, Aluminiumsubstrate is placed in $2 \mathrm{M}$ suphuric acid for 30 minutes it shows WCA of $162.0^{\circ}$ (for $180 \mathrm{~min}$ ) which is a superhydrophobic surface Figure $3 \mathrm{~d}$.
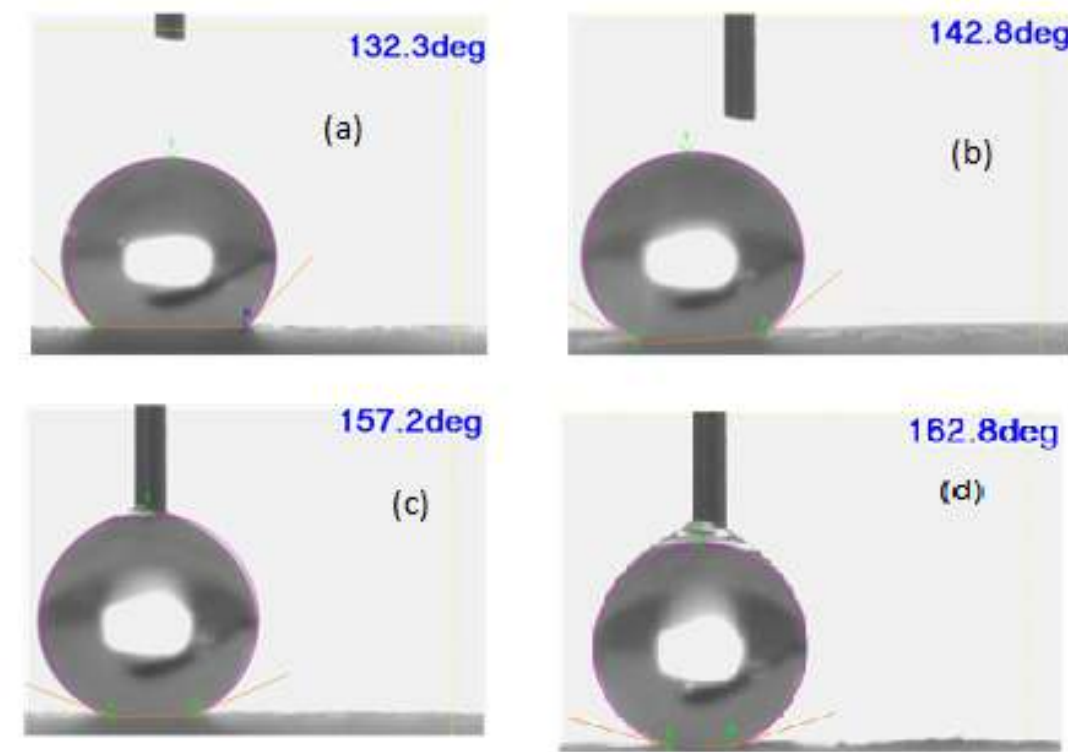

Figure 3: The optical image of WCA of treated aluminium material (a) ASH1, (b) ASH2, (c) ASH3 and (d) ASH4.

\subsection{Surface morphology}

The surface morphology of the chemically etched aluminium substrate, anodized and sealed aluminium substrate and superhydrophobicaluminium substrate are shown in the Figure 4. The microscopic defects are clearly seen in the bare aluminium substrate Figure 4 a. After anodizing and sealing the micro nano structure is developed on the aluminium substrate $4 \mathrm{~b}$. These microscopic defects on the aluminium surface is formed higher energy. Figure $4 \mathrm{c}$ represents the formation of superhydrophobic surface on the aluminium substrate, the micronano structure of the anodized aluminium substrate were covered by the longer hydrocarbon chain of lauric acid, the projecting groups are responsible for the superhydrophobic nature of the fabricated substrate (ASH4).[20] 

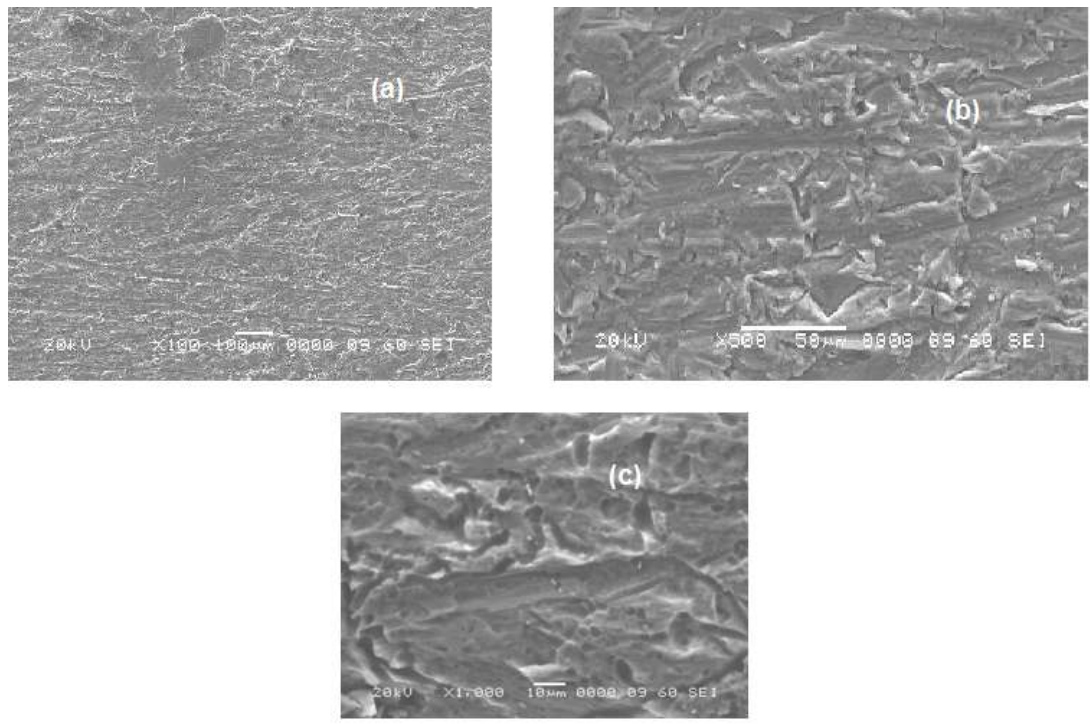

Figure 4: SEM images of (a) chemically etched aluminium substrate, (b) anodized and sealed aluminium substrate and (c) ASH4

\subsection{Corrosion analysis of superhydrophobic surface}

Resistance to corrosion against an aggressive corrosion media is one of the important phenomena to measure the wettability phenomenon to test the stability of superhydrophobic surfaces. Greater the corrosion resistance greater will be the stability of the superhydrophobic coating. Tafel experiments are carried out using three electrode set up where, saturated calomel electrode acts as reference electrode, platinum foil is used as counter electrode and the untreated aluminium plate/ASH4 is used as working electrode. The corrosion measurements are done using Gamry reference 600 potentiostat. The data were analyzed by the GamryEchem analyst software. The Tafel plots for the bare aluminium and ASH4 are given in the Figure 5 


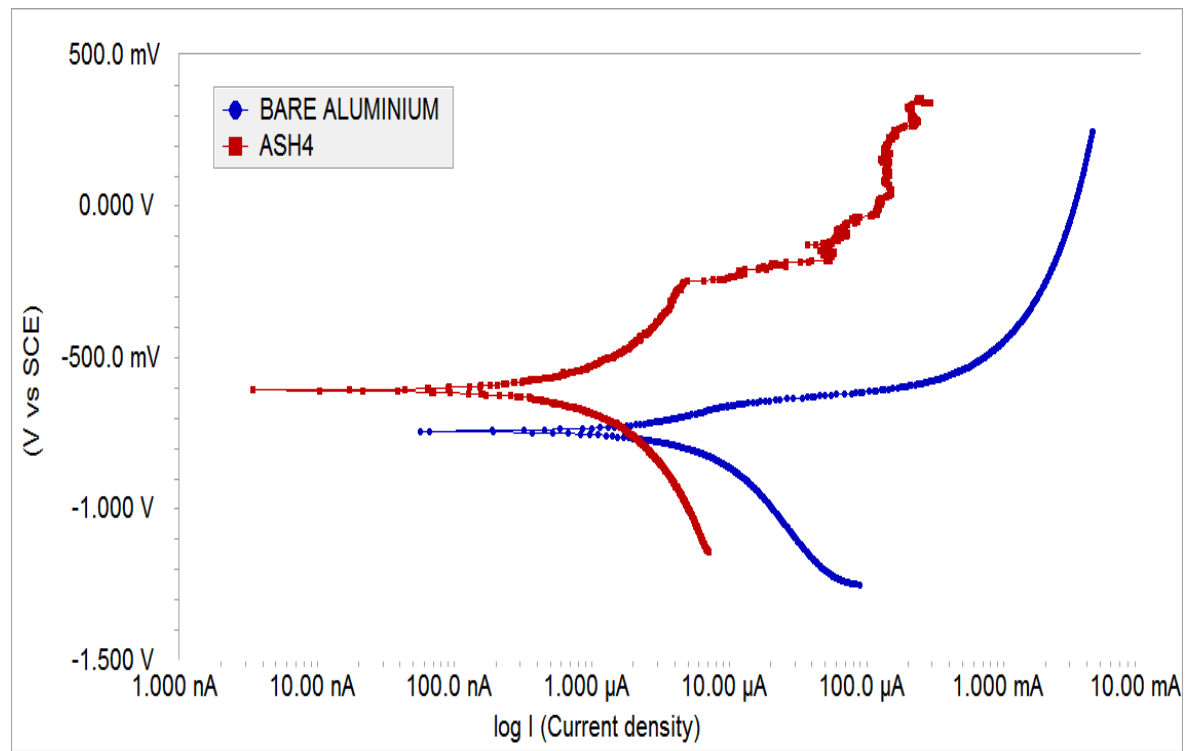

Figure 5: Potentiodynamic polarization Tafel plots for the corrosion bare aluminium and ASH4 in $3.5 \% \mathrm{NaCl}$ solution

The potentiodynamic polarization data were represented in the Table 2The Corrosion protection efficiency of the ASH4 over the bare aluminium substrate is calculated using the following formula.[21-23]

Corrosion protection efficiency (\%)

$$
=\left(100-\frac{(\text { CR of bare aluminium }- \text { CR of SH aluminium })}{\text { CR of bare aluminium }}\right)-- \text { eq. } 7
$$

\begin{tabular}{|l|l|l|l|l|l|}
\hline Medium & $\begin{array}{l}\text { Name of the } \\
\text { sample }\end{array}$ & $\begin{array}{l}\text { Icorr } \\
(\mathbf{m A})\end{array}$ & $\begin{array}{l}\text { Ecorr } \\
\mathbf{( m V )}\end{array}$ & $\begin{array}{l}\text { Corrosion } \\
\text { rate (mpy) }\end{array}$ & $\begin{array}{l}\text { Corrosion } \\
\text { protection } \\
\text { efficiency (\%) }\end{array}$ \\
\hline \multirow{2}{*}{$\begin{array}{l}3.5 \% \mathrm{NaCl} \\
\text { solution }\end{array}$} & $\begin{array}{l}\text { Bare } \\
\text { Aluminium }\end{array}$ & $\begin{array}{l}7.120 \mathrm{x} \\
10^{3}\end{array}$ & -743.0 & 9.165 & - \\
\cline { 2 - 6 } & ASH4 & $\begin{array}{l}1.570 \mathrm{x} \\
10^{3}\end{array}$ & -607.0 & 2.027 & 99.2 \\
\hline
\end{tabular}

Table 2: Electrochemical polarization parameters recorded during corrosion of bare aluminium and $\mathrm{ASH} 4$ in $3.5 \% \mathrm{NaCl}$ solution.

From the table it is clearly seen that the fabricated superhydrophobic surface shows very high corrosion resistance of about $99 \%$. The reason behind the higher corrosion resistance of the ASH4 are as follows: i) the anodized oxide layer imparts good corrosion resistance, ii) the longer chain hydrocarbon chain of lauric acid may be projecting out of the aluminiumsubstrate, which minimizes the interaction between the water and metallic substrate. Since the interaction between water and metal is minimized the possibilities of 
corrosion also decreases. The effectiveness of the superhydrophobic surface were clearly visible from the static WCA of the ASH4. As we know, greater the WCA the hydrophobicity increases and the metal water interaction decreases which ultimately decreases the corrosion of the base material.

\section{Conclusion}

A simplistic method to obtain super hydrophobic surface on aluminium is successfully designed. The following conclusion are:

- The fabricated superhydrophobic surface shows very good non-wetting behavior.

- The static WCA of fabricated surface confirms that the fabricated surface is of highly superhydrophobic in nature.

- The anodized layer act as bridge between aluminum and superhydrophobic mixture and also increases the mechanical and corrosion resistance of the substrate.

- The surface morphology analysis using SEM conforms the projection of sodium laurate group.

- By adopting this simple procedure any type of alminium substrate can be converted to superhydrophobic in nature.

\section{References}

[1] M. Ma, R.M. Hill, Superhydrophobic surfaces, Current opinion in colloid \& interface science, 11 (2006) 193-202.

[2] X. Zhang, F. Shi, J. Niu, Y. Jiang, Z. Wang, Superhydrophobic surfaces: from structural control to functional application, Journal of Materials Chemistry, 18 (2008) 621-633.

[3] X.-M. Li, D. Reinhoudt, M. Crego-Calama, What do we need for a superhydrophobic surface? A review on the recent progress in the preparation of superhydrophobic surfaces, Chemical Society Reviews, 36 (2007) 1350-1368.

[4] E. Celia, T. Darmanin, E.T. de Givenchy, S. Amigoni, F. Guittard, Recent advances in designing superhydrophobic surfaces, Journal of colloid and interface science, 402 (2013) 1-18.

[5] J.P. Rothstein, Slip on superhydrophobic surfaces, Annual review of fluid mechanics, 42 (2010) 89-109.

[6] A. Carré, K.L. Mittal, Superhydrophobic surfaces, CRC Press, 2009.

[7] A. Marmur, The lotus effect: superhydrophobicity and metastability, Langmuir, 20 (2004) 35173519.

[8] L. Gao, T.J. McCarthy, The "lotus effect" explained: two reasons why two length scales of topography are important, Langmuir, 22 (2006) 2966-2967.

[9] M. Zhang, S. Feng, L. Wang, Y. Zheng, Lotus effect in wetting and self-cleaning, Biotribology, 5 (2016) 31-43.

[10] A. Kozbial, C. Trouba, H. Liu, L. Li, Characterization of the intrinsic water wettability of graphite using contact angle measurements: effect of defects on static and dynamic contact angles, Langmuir, 33 (2017) 959-967.

[11] C. Huang, S.-Y. Wu, Y.-C. Liu, Y.-C. Chang, C.-Y. Tsai, Static water contact angle analysis of cyclonic atmospheric pressure plasma-activated polycarbonate, Japanese Journal of Applied Physics, 50 (2011) 01AH05.

[12] F.M. Helmi, Y.K. Hefni, A simple method for measuring the static water contact angle for evaluation the hydrophobicity of the consolidating and protective materials, (2014).

[13] D. Kwok, A.W. Neumann, Contact angle interpretation in terms of solid surface tension, Colloids and Surfaces A: Physicochemical and Engineering Aspects, 161 (2000) 31-48. 
[14] N. Adam, Use of the term 'Young's Equation'for contact angles, Nature, 180 (1957) 809-810.

[15] R.N. Wenzel, Surface roughness and contact angle, The Journal of Physical Chemistry, 53 (1949) 1466-1467.

[16] A. Cassie, S. Baxter, Wettability of porous surfaces, Transactions of the Faraday society, 40 (1944) 546-551.

[17] A. Cassie, Contact angles, Discussions of the Faraday society, 3 (1948) 11-16.

[18] R. Hinde, E. Kellett, P. Harris, Sealing of aluminium oxide anodic films, Nature, 183 (1959) 39-39.

[19] G.D. Sulka, K.G. Parkoła, Anodising potential influence on well-ordered nanostructures formed by anodisation of aluminium in sulphuric acid, Thin Solid Films, 515 (2006) 338-345.

[20] T. Nakanishi, Y. Shen, J. Wang, H. Li, P. Fernandes, K. Yoshida, S. Yagai, M. Takeuchi, K. Ariga, D.G. Kurth, Superstructures and superhydrophobic property in hierarchical organized architectures of fullerenes bearing long alkyl tails, Journal of Materials Chemistry, 20 (2010) 1253-1260.

[21] K. Mallaiya, R. Subramaniam, S.S. Srikandan, S. Gowri, N. Rajasekaran, A. Selvaraj, Electrochemical characterization of the protective film formed by the unsymmetrical Schiff's base on the mild steel surface in acid media, Electrochimica Acta, 56 (2011) 3857-3863.

[22] S.H.M. Jessima, S. Subhashini, A. Berisha, A. Oral, S.S. Srikandan, Corrosion mitigation performance of disodium EDTA functionalized chitosan biomacromolecule-Experimental and theoretical approach, International Journal of Biological Macromolecules, 178 (2021) 477-491.

[23] PM. Tholkapiyan, A.Mohan, Vijayan.D.S , "A survey of recent studieson chlorophyll variation in Indian coastal waters", IOP Conf. Series: Materials Science and Engineering 993 (2020) 012041, doi:10.1088/1757-899X/993/1/012041. 